\title{
Small Elevations of Glucose Concentration Redirect and Amplify the Synthesis of Guanosine 5'-Triphosphate in Rat Islets
}

\author{
Stewart A. Metz, Melissa Meredith, Mary E. Rabaglia, and Anjaneyulu Kowluru \\ Department of Medicine and Division of Endocrinology, University of Wisconsin, Madison, Wisconsin 53792; \\ and Middleton Memorial Veterans Medical Center, Madison, Wisconsin 53705
}

\begin{abstract}
Recent studies suggest a permissive requirement for guanosine 5 '-triphosphate (GTP) in insulin release, based on the use of GTP synthesis inhibitors (such as mycophenolic acid) acting at inosine monophosphate (IMP) dehydrogenase; herein, we examine the glucose dependency of GTP synthesis. Mycophenolic acid inhibited insulin secretion equally well after islet culture at 7.8 or $11.1 \mathrm{mM}$ glucose ( $51 \%$ inhibition) but its effect was dramatically attenuated when provided at $\leq 6.4 \mathrm{mM}$ glucose (13\% inhibition; $P<0.001)$. These observations were explicable by a stimulation of islet GTP synthesis derived from IMP since, at high glucose: $(a)$ total GTP content was augmented; (b) a greater decrement in GTP (1.75 vs. $1.05 \mathrm{pmol}$ / islet) was induced by mycophenolic acid; and (c) a smaller "pool" of residual GTP persisted after drug treatment. Glucose also accelerated GTP synthesis from exogenous guanine ("salvage" pathway) and increased content of a pyrimidine, uridine 5 '-triphosphate (UTP), suggesting that glucose augments production of a common regulatory intermediate (probably 5phosphoribosyl-1-pyrophosphate). Pathway-specific radiolabeling studies confirmed that glucose tripled both salvage and de novo synthesis of nucleotides. We conclude that steep changes in the biosynthesis of cytosolic pools of GTP occur at modest changes in glucose concentrations, a finding which may have relevance to the adaptive ( patho) physiologic responses of islets to changes in ambient glucose levels. (J. Clin. Invest. 1993. 92:872-882.) Key words: (Pancreatic) islet • nucleotide • purine $\bullet$ adenosine triphosphate $\bullet$ guanine

\section{Introduction}

We recently reported that exocytotic insulin secretion from isolated, intact rat pancreatic islets depends upon an adequate islet content of guanosine $5^{\prime}$-triphosphate $(\mathrm{GTP})^{1}(1)$.

Portions of this work were presented at the 52nd Annual Meeting of the American Diabetes Association, San Antonio, TX, June 21, 1992 (1992. Diabetes. 41 [Suppl.]:3a); the Midwest Section of the American Federation for Clinical Research, Chicago, IL, November 6, 1992 (1992. Clin. Res. 40:734a[Abstr.]); and the 28th Annual Meeting of the European Association for the Study of Diabetes, Prague, Czechoslovakia, September 8, 1992 (1992. Diabetologia. 35:[Suppl.]A116).

Address correspondence to Dr. Stewart A. Metz, Division of Endocrinology, University of Wisconsin Medical School (H4/554 Clinical Science Center), 600 Highland Avenue, Madison, WI 53792.

Received for publication 1 December 1992 and in revised form 24 March 1993.

1. Abbreviations used in this paper: CTP, cytidine 5'-triphosphate; Gln, L-glutamine; GTP, guanosine 5'-triphosphate; HGPRTase, hypoxan-

The Journal of Clinical Investigation, Inc.

Volume 92, August 1993, 872-882
The conclusion was based on the use of four selective inhibitors of inosine monophosphate (IMP) dehydrogenase (EC 1.1.1.205), a rate-limiting step in the de novo synthetic pathway for purine nucleotides. Two of these drugs, mycophenolic acid (MPA) and mizoribine, when provided during an 18-h culture period in RPMI 1640 (a purine-free medium), were shown to drastically curtail islet content of GTP and, pari passu, the subsequent insulin secretory responses to glucose and other secretagogues. MPA or mizoribine also caused a more modest inhibition of ATP content (by about $40 \%$ ) and dramatic elevations of a pyrimidine, uridine 5'-triphosphate (UTP), presumably due to redirection of a precursor common to both pyrimidine and purine synthesis, such as 5-phosphoribosyl-1-pyrophosphate (PRPP). However, GTP was implicated as the regulatory factor since: $(a)$ provision of exogenous adenine reversed changes in ATP and UTP, while leaving unaltered the decrements in GTP and in insulin release; and $(b)$ provision of exogenous guanine, by fueling the "salvage" pathway for purine synthesis, restored both GTP and secretion nearly to normal levels. We concluded therefore that GTP was a permissive factor required for exocytotic insulin release (1).

In those studies, pancreatic islets were cultured overnight in $11.1 \mathrm{mM}(200 \mathrm{mg} / \mathrm{dl})$ of glucose, since this usually is the most effective concentration for cultured islets to maintain insulin content and secretion $(2,3)$. However, we were intrigued by an absence of reports of the induction of hyperglycemia in normal animals or humans treated with high concentrations of these drugs (e.g., MPA, provided as its pro-drug RS-61443, references 4 and 5). Therefore, we questioned whether the ambient glucose concentration might modulate the islet content of purine nucleotides and, if so, whether glucose might alter the relative predominance of the pathways involved in the synthesis of GTP. The current studies (the first to our knowledge which address purine nucleotide metabolic pathways in peptide-secreting endocrine cells) provide data that support both possibilities and suggest that glucose alters purine nucleotide metabolism in both quantitative and qualitative fashions. The narrow range of relevant glucose concentrations over which such responses can be discerned in pancreatic islets suggests that these events could have (patho)physiologic relevance to some of the islet's nucleotide-dependent adaptive responses to hyperglycemia, such as insulin secretion (1), RNA and protein synthesis (6), and DNA synthesis (7).

\section{Methods}

Materials. Mycophenolic acid, nucleotide standards, monobasic ammonium phosphate (for HPLC mobile phase) and guanine were pur-

thine-guanine phosphoribosyltransferase; IMP, inosine monophosphate; ITP, inosine 5'-triphosphate; MPA, mycophenolic acid; NTP, nucleoside triphosphate; PRPP, 5-phosphoribosyl-1-pyrophosphate; UTP, uridine 5'-triphosphate; XMP, xanthosine monophosphate. 
chased from Sigma Chemical Co., (St. Louis, MO). Mizoribine, also referred to as bredinin (4-carbamoyl-1- $\beta$-D-ribofuranosylimidazolium5-olate; references 8-10) was a generous gift of Dr. N. Kazmatani ( Tokyo Women's Medical College, Tokyo). The diluents used for making stock solutions of these drugs were ethanol (for mycophenolic acid), water (for mizoribine), and $\mathrm{Me}_{2} \mathrm{SO}$ (for guanine), respectively; control tubes always contained an equal amount of the relevant diluent as experimental tubes. $\left[{ }^{3} \mathrm{H}(\mathrm{G})\right]$ hypoxanthine $(17 \mathrm{Ci} / \mathrm{mmol})$ and $\left[{ }^{14} \mathrm{C}(\mathrm{U})\right]$ glycine $(110.5 \mathrm{mCi} / \mathrm{mmol})$ were purchased from New England Nuclear (Boston, MA) or Amersham Corp. (Arlington Heights, IL). HPLC columns were purchased from Alltech Associates, Inc. (Deerfield, IL); HPLC water was purchased from Fisher Scientific (Itasca, IL). RPMI 1640 medium was purchased from Gibco (Grand Island, NY) as glucose-free powder to which the desired amount of D-glucose was added to achieve glucose concentrations of 1.7 to 11.1 $\mathrm{mM}$ in the overnight culture medium. L-glutamine (Gln, $2.05 \mathrm{mM}$ ) was present in the culture medium to provide adequate substrate for the de novo synthesis of purine nucleotides (11-14); where indicated, glycine content was selectively modified using the Select-Amine Kit from Gibco. Undialyzed FBS was from Gibco. In all studies comparing the effects of two different glucose concentrations during the culture period, the osmolarity of the medium containing the lower glucose concentration was brought to that of the higher concentration through the provision of additional $\mathrm{NaCl}$.

Isolation and treatment of pancreatic islets for studies of insulin release. Intact pancreatic islets (usually 500-600 islets per study) were isolated from adult male Sprague-Dawley rats (Sasco-King, Inc., Omaha, NE) using collagenase digestion and separation from acinar tissue and debris on ficoll gradients, followed by hand-picking under stereomicroscopic control, as previously described $(1,15-17)$. Islets were cultured overnight $(\times 18 \mathrm{~h})$ in RPMI 1640 medium (containing $10 \% \mathrm{FBS}, 100 \mathrm{U} / \mathrm{ml}$ penicillin, $100 \mu \mathrm{g} / \mathrm{ml}$ of streptomycin, and variable glucose concentrations). Generally speaking, each secretion experiment contained 4-8 experimental conditions, each comprised of multiple ( 3 to 8 ) replicate determinations ( 10 islets each). The incubation medium for subsequent insulin release studies (carried out the next day) was $\mathrm{KRB}$, pH 7.4, containing $0.5 \%$ BSA and 3.3 or $16.7 \mathrm{mM}$ glucose, and gassed with $95 \% \mathrm{O}_{2} / 5 \% \mathrm{CO}_{2}$. Static, batch-type incubations were carried out for 45 mins so as to include contributions from both first and second phase secretion, since our previous studies (1) indicated that MPA inhibits both phases of glucose-induced insulin release. The basal glucose concentration for acute incubations (i.e., after the overnight culture period ) was $3.3 \mathrm{mM}$; the glucose concentration used to stimulate islets in all incubations was kept constant at 16.7 mM. MPA (or other test agents) was present during not only the culture period but also was included in islet picking, wash, or preincubation steps the following day, in order to avoid attenuation of its effects over time. However, drugs were excluded from the incubation period, since their effects were not rapidly dissipated after removal (1).

Insulin content and fractional release. After incubations to assess insulin release, the tubes containing islets, still resting on their polyethylene filters (15), were placed in $15-\mathrm{ml}$ conical centrifuge tubes containing $1 \mathrm{ml}$ of acid alcohol (comprised of $77 \%$ absolute ethanol, $22 \%$ water, and $1 \%$ concentrated $\mathrm{HCl}$; vol/vol), sonicated and left overnight at $4^{\circ} \mathrm{C}$. After $20 \mathrm{~h}$, one $\mathrm{ml}$ of phosphate-buffered saline and $30 \mu \mathrm{l}$ of $5 \mathrm{~N} \mathrm{NaOH}$ were added to raise the $\mathrm{pH}$ to 8.0 , and the contents were vortexed after removing the glass tube but leaving behind the meshfilter so as to retain any islet tissue which might remain adherent to the filter. Tubes were centrifuged at $4^{\circ} \mathrm{C}$ for $5 \mathrm{~min}(\times 2,000 \mathrm{rpm})$; the supernatant was removed, diluted (1:10) in RIA buffer (PBS) to a final dilution of $1: 20$, and assayed.

Insulin content of media or of islets was measured by RIA as described (1, 15-17).

Determination of nucleotide content of islets. Islets were cultured overnight (as described above and in reference 1) except that exactly 200 islets for each condition were cultured in individual dishes containing test substances, so that islets could be rapidly extracted the next day without the need for time-consuming counting and aliquoting, during which the nucleoside triphosphate (NTP) contents might change. A typical experiment contained 12 determinations comprised of 2,400 islets/study; three to four experimental conditions ( each in triplicate or quadruplicate) were studied in each experiment. After the culture period, islets were treated as previously described in detail (1) and were extracted in trichloroacetic acid (TCA), containing sufficient internal standard cytidine 5'-triphosphate (CTP) and inosine 5'-triphosphate (ITP) to yield a final concentration of $0.8 \mathrm{nmol} / 100 \mu \mathrm{l}$. Islets were then lightly vortexed, sonicated $(2 \times 20 \mathrm{~s})$ on ice, and the supernatant was obtained after centrifugation, all as described (1). Supernatants were extracted ( $\times 4)$ using $0.75 \mathrm{ml}$ ether (to remove the TCA); the ether phase was then discarded. Samples were directly analyzed by HPLC or frozen at $-20^{\circ} \mathrm{C}$ for future analysis within two weeks; there was no difference in results between the two handling procedures.

HPLC was carried out as described (1) using a LiChrosorb- $\mathrm{NH}_{2}$ anion exchange column (Alltech Associates, Inc.) without a guard column. The detector was set at $254 \mathrm{nM}$ and 0.02 to 0.005 AUFS. Buffer A was $0.5 \mathrm{mM} \mathrm{NH}{ }_{4} \mathrm{H}_{2} \mathrm{PO}_{4}$, $\mathrm{pH} \mathrm{2.65}$; buffer $\mathrm{B}$ was $0.65 \mathrm{M} \mathrm{NH}_{4} \mathrm{H}_{2} \mathrm{PO}_{4}$, $\mathrm{pH}$ 2.65. The exact proportions of $\mathrm{A}$ and $\mathrm{B}$ and the $\mathrm{pH}$ were varied as described in (1) using a solvent programmer (Model 660; Waters Instruments, Inc., Milford, MA ) in order to maximize separations, which were affected by changes in columns or new batches of solvent, and, especially, by even minor changes in $\mathrm{pH}$. Separations were achieved by isocratic elution at $1.0-1.3 \mathrm{ml} / \mathrm{min} .100 \mu \mathrm{l}$ each of sample (comprising the extract of $\sim 70$ islets) was injected in duplicate; results were corrected for the mean recovery of two exogenous nucleotides (ITP and CTP, which were used as internal standards after preliminary studies ascertained that they were not detectable endogenously in islet extracts). A standard curve was routinely run each day, covering the relevant range $(0.2-1.0 \mathrm{nmol})$ of ITP, CTP, GTP, ATP, and UTP. Values for the recoveries of internal standards (ITP, CTP) have been reported, as have been the coefficients of variation for the overall precision of the measurements (1). Values for basal islet nucleotide content were sufficiently constant (inter-experiment CVs of $6.8,7.2$, and $10.8 \%$ for basal ATP, GTP, and UTP content, respectively) as to permit meaningful comparisons of the effect of experimental perturbations not only within, but also between, studies.

Labeling of purine nucleotides via the de novo or salvage pathways. In order to determine whether the de novo or salvage pathways contributed to the total GTP or ATP content, islets were labeled with 10 $\mu \mathrm{Ci} / \mathrm{ml}$ of $\left[{ }^{14} \mathrm{C}(\mathrm{U})\right]$ glycine (which donates carbons at position 4 and 5 , and nitrogen at position 7 , of the newly-synthesized purine ring) or with $2 \mu \mathrm{Ci} / \mathrm{ml}$ of $\left[{ }^{3} \mathrm{H}(\mathrm{G})\right]$ hypoxanthine (a substrate for HGPRTase) over an identical 18 -h period. The added mass of hypoxanthine was kept low $(\sim 120 \mathrm{nM})$, and labeled glycine replaced an equal mass of unlabeled glycine in the RPMI 1640 media, so as not to perturb the conditions under which ATP/GTP had been assessed by mass in the preceding studies; in addition, all other experimental conditions remained unchanged. After $18 \mathrm{~h}$, islets were extracted and analyzed by HPLC as indicated (1) except that an additional rapid wash in $1 \mathrm{ml}$ $\mathrm{KRB}$ buffer containing $0.5 \% \mathrm{BSA}$ was added in order to remove more completely any unincorporated radiolabeled compounds adherent to the islets. Eluant fractions were collected every $30 \mathrm{~s}$ (glycine label) or every $18 \mathrm{~s}$ (hypoxanthine label) using a fraction collector (Multi-rac 211; LKB Instruments, Bromma, Sweden ) and were matched against the exact times of elution of GTP and ATP using the concomitant UV chromatograms. Samples were counted for $5 \mathrm{~min}$ in $4 \mathrm{mls}$ of scintillation cocktail.

Data presentation and statistical analysis. Absolute insulin secretion is expressed as $\mu \mathrm{U} / 10$ islets .45 min (mean \pm SEM) for static incubations. Incremental release is expressed as the value for insulin released under stimulated conditions (minus) the mean value for basal release (i.e., at $3.3 \mathrm{mM}$ glucose) in that same study. Insulin content is expressed as $\mu \mathrm{U} /$ islet remaining at the end of the incubation period. Some fractional release rates are also presented to take into account any effects on absolute rates of secretion caused by alterations in islet insulin content induced by agents such as MPA (1). Fractional release was calculated by the formula: 
fractional release $=\left[\frac{\text { insulin released }}{\text { initial insulin content }}\right]$,

where the denominator equals the insulin content remaining in islets at the end of the incubation period plus that insulin released during the incubation period. Fractional release is expressed as percent of initial insulin content released per 45 mins.

Nucleotide content is expressed as pmol/islet; specific activity is expressed as $\mathrm{dpm} / \mathrm{pmol}$. Increments or decrements in nucleotide content are expressed as the content of each experimental tube (minus) the mean content of the control tubes in that study; alternatively, experimental values are expressed in terms of percent of their mean control values ( the glucose concentration used to define the "control" response for each experiment is indicated in the text or figure legends). Data are expressed as mean \pm SEM, with $(n)$ representing the number of observations in a representative study, or the number of separate experiments, as indicated. Statistical analyses were by paired or nonpaired $t$ tests, and ANOVA, as appropriate.

\section{Results}

Dependency on glucose concentration of the inhibition by MPA of glucose-induced insulin release. Glucose concentrations from $50-200 \mathrm{mg} / \mathrm{dl}(2.8-11.1 \mathrm{mM})$ were chosen for the overnight culture period. $25 \mu \mathrm{g} / \mathrm{ml}$ of MPA was used in all studies since it induces a maximal inhibition of GTP synthesis (1). It was confirmed (1) that an overnight exposure to MPA inhibits subsequent insulin release induced by $16.7 \mathrm{mM}$ glucose; however, the inhibitory effect of MPA was influenced dramatically by the glucose concentration present during the preceding $18-\mathrm{h}$ culture period (Fig. 1). Islets cultured at $7.8 \mathrm{mM}$ glucose were at least as sensitive to MPA as were islets cultured at $11.1 \mathrm{mM}$ glucose, but sensitivity to MPA declined steeply at lower glucose concentrations, being half-maximal at $6.4 \mathrm{mM}$ glucose, and essentially absent at $4.4 \mathrm{mM}$ glucose (Fig. 1). In paired analysis of data accrued within the same experiments, inhibition was always greater when MPA was provided at 7.8-11.1 $\mathrm{mM}$ glucose $(-51 \pm 6 \%)$ than at $\leq 6.4 \mathrm{mM}(-13 \pm 6 \% ; P$ $<0.001$ for six paired glucose-concentration comparisons within five separate experiments, as depicted by individual symbols in the inset to Fig. 1). In our cumulative experience using MPA (including data in reference 1), there was no overlap in the range of inhibition by MPA at higher glucose levels (range: 34 to $86 \% ; n=34$ separate experiments) and that at lower glucose level (range: 0 to $34 \% ; n=6 ; P<0.001$ by nonpaired $t$ test). Such comparisons underestimate the difference at higher $v s$. lower glucose concentrations, since preexposure to MPA at $4.4 \mathrm{mM}$ glucose actually augmented subsequent insulin release in two experiments; these values are treated as "zero inhibition" for statistical purposes. It is not likely that the findings with MPA represent a aberration specific to that pharmacologic probe, since a second, selective inhibitor of inosine monophosphate dehydrogenase $(75 \mu \mathrm{g} / \mathrm{ml}$ mizoribine) did not inhibit subsequent insulin release at all when provided at $4.4 \mathrm{mM}$ glucose (data not shown), in contradistinction to an inhibition of $47 \%$ (absolute release) and of $52 \%$ (fractional release), seen after $11.1 \mathrm{mM}$ glucose.

These results might be explicable if MPA were to exert a glucose-dependent effect on insulin content of islets. As previously observed (1), MPA did, in fact, reduce insulin content after culture in high glucose (by $20 \pm 4 \%$ ) whereas after expo-

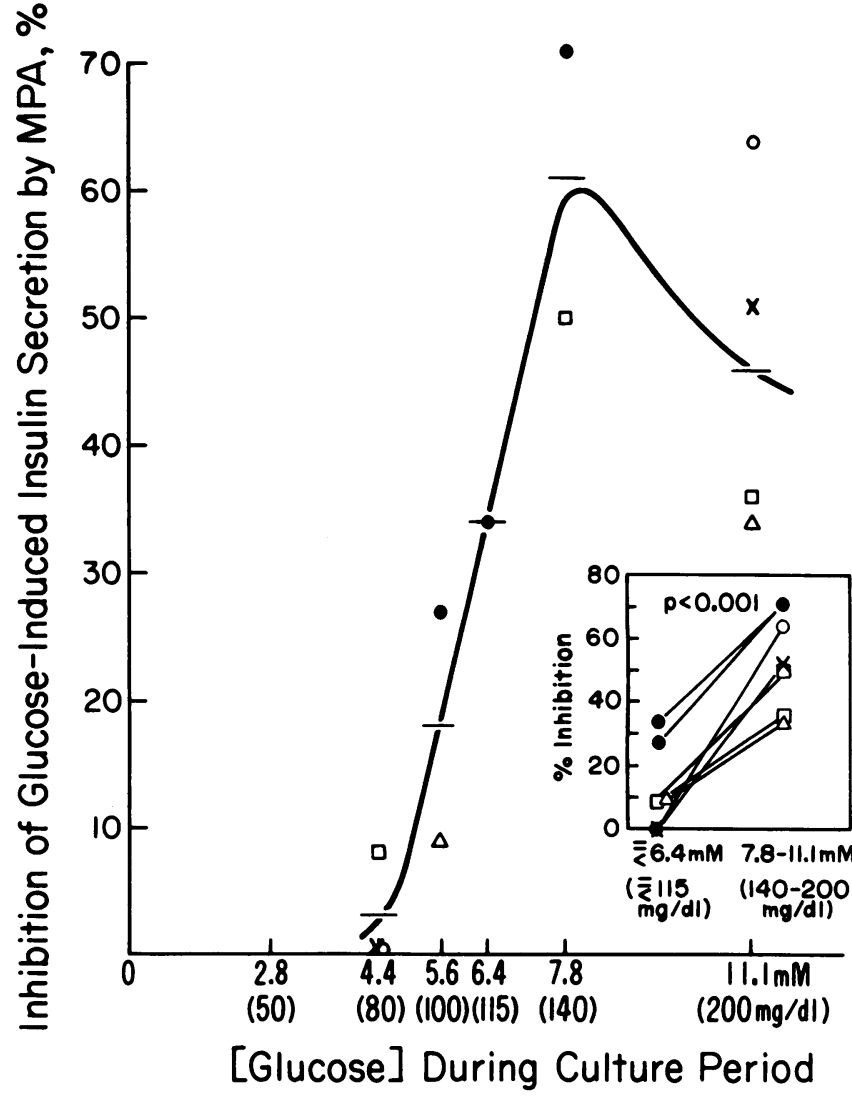

Figure 1. Glucose dependency of MPA inhibition of insulin secretion. The glucose concentration during the preceding 18-h culture period is expressed on the abscissa and the inhibition by MPA of the subsequent incremental insulin secretory response to a maximal glucose ( $16.7 \mathrm{mM}$ ) stimulus is presented on the ordinate. Each symbol comprises the mean of multiple (3-8) determinations carried out after culture at the indicated glucose concentration on a single day, with each symbol representing a single such experiment. Identical symbols indicate findings within a single experiment. The horizontal lines in the main panel indicate the mean response at each glucose concentration. Statistical analysis (by paired $t$ test) is presented in the inset, in which a dividing point of $6.4 \mathrm{mM}$ glucose was used to analyze responses to higher vs. lower glucose concentrations during culture. Paired responses within the same study are connected by solid lines.

sure of islets to lower glucose levels, MPA reduced insulin content by only $7 \pm 3 \%(n=5$ experiments; $P<0.01$; Table I $)$. In fact, at $2.8 \mathrm{mM}$ glucose, MPA actually increased insulin content (Table I). However, the effects of MPA on exocytotic release were independent of these effects of MPA on insulin content, since fractional insulin release (insulin release/insulin content) was also reduced much less by MPA during culture of islets at lower glucose levels $(-14 \pm 7 \% ; P=$ ns $v s$. no MPA) than after culture at $7.8-11.1 \mathrm{mM}$ glucose $(-44 \pm 5 \% ; n=5$ experiments; $P<0.0001 v s$. no MPA; $P<0.01 v s$. inhibition by MPA at lower glucose levels; Table I).

Effects of glucose on GTP and ATP content, and their dependency on MPA-inhibitable pools. In order to explain the glucose dependency of MPA effects on secretion, the nucleotide content of islets was analyzed using a similar experimental paradigm. In addition, we estimated the proportion of the steady-state GTP (and ATP) content that was derived ultimately from IMP, by taking advantage of the facts that MPA is 
Table I. Insulin Content ( $\mu$ U/10 islets) of Pancreatic Islets, and Fractional Insulin Release (\% 45 min), in the Presence and Absence of Prior Exposure $(\times 18$ h) to MPA at Lower vs. Higher Glucose Concentrations *

4.4-6.4 mM glucose $\quad 7.8-11.1 \mathrm{mM}$ glucose $P$

A. Insulin content ${ }^{\ddagger}$

(-) MPA

(+) MPA

B. Fractional insulin release

$(-)$ MPA

$(+)$ MPA

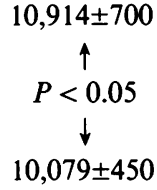

$3.35 \pm 0.91$
$\uparrow$
$\mathrm{NS}$
$\downarrow$
$3.11 \pm 0.49$

$$
\begin{gathered}
8,009 \pm 1,016 \\
\uparrow \\
P<0.01 \\
\downarrow \\
6,282 \pm 628
\end{gathered}
$$

$$
\begin{gathered}
6.05 \pm 0.54 \\
\uparrow \\
P<0.0001 \\
\downarrow \\
3.37 \pm 0.35
\end{gathered}
$$

NS

\footnotetext{
* Data are mean $( \pm$ SEM) for five separate experiments comprised of six sets of paired data at different glucose concentrations (as indicated in legend to Fig. 1). $\quad{ }^{\ddagger}$ In an additional experiment, MPA reduced insulin content by $30 \%$ at $11.1 \mathrm{mM}$ glucose (control: $8,359 \pm 756, n=7 ;$ MPA: $5,872 \pm 394, n=7, P<0.01$ ) whereas insulin content actually rose by $20 \%$ in the presence of MPA at $2.8 \mathrm{mM}$ glucose (control: $10,459 \pm 498, n$ $=8$; MPA: $12,504 \pm 808, n=8 ; P<0.025$ ). The control insulin secretory response to glucose was too low for the effect of MPA to be interpretable in this experiment.
}

a selective inhibitor of GTP synthesis at the level of IMP dehydrogenase (18-22) with no direct effects on enzymes of the "salvage" (or other) pathways $(20,21,23,24)$. The contribution of IMP-dependent pools at each glucose level was defined as the decrement in GTP induced by a maximally-effective concentration of MPA (25 $\mu \mathrm{g} / \mathrm{ml}$; reference 1 ), whereas the contribution of IMP-independent pathways to steady-state GTP content was estimated as the amount of GTP resistant to blockade by MPA. Additionally, the increment in GTP concentration induced by $75 \mu \mathrm{M}$ of guanine, provided exogenously, was taken as a measure of net salvage pathway activity (i.e., reflecting hypoxanthine-guanine phosphoribosyltransferase activity).

Culturing islets at $11.1 \mathrm{mM}$ glucose reproducibly increased their content of both GTP (by 24 to 33\%) and ATP (by 13 to $20 \%$ ) above values seen at $4.4 \mathrm{mM}$ glucose; UTP also rose (see below) (Figs. 2-5). Therefore, total NTP content also increased by $33 \%$ from $13.77 \pm 0.79$ to $18.38 \pm 1.24 \mathrm{pmol} /$ islet ( $\mathrm{df}$ $11 ; P<0.01)$. These effects were not explicable by a nonspecific (e.g., osmotic) effect of hexoses, since osmotic controls were present in all studies (see Methods); furthermore, the addition to $4.4 \mathrm{mM}$ glucose of $6.7 \mathrm{mM} 3-0$-methylglucose (which is transported similarly to glucose but which is not metabolized; reference 25 ) actually slightly inhibited (by 5, 6, and $10 \%$, respectively) content of GTP, ATP, and UTP (data not shown ). Furthermore, the NTP content of islets declined if the glucose level was reduced from 4.4 to $1.7 \mathrm{mM}$ glucose (Table II); it fell to a comparable degree when $3.0 \mathrm{mM}$ of mannoheptulose (which blocks the phosphorylation and further metabolism of glucose; reference 26 ) was added to $4.4 \mathrm{mM}$ glucose during culture (Table II).

Additionally, MPA reduced GTP or ATP content more at $11.1 \mathrm{mM}$ glucose than at $4.4 \mathrm{mM}$ glucose (both $P<0.001$; Figs. 2, 3, and 5). Thus, despite starting from higher levels at
$11.1 \mathrm{mM}$ glucose, MPA reduced GTP content by a greater amount and to lower absolute levels at high glucose (Figs. 2 and 5). Furthermore, during MPA treatment, glucose no longer increased GTP (or ATP) content; rather, the residual

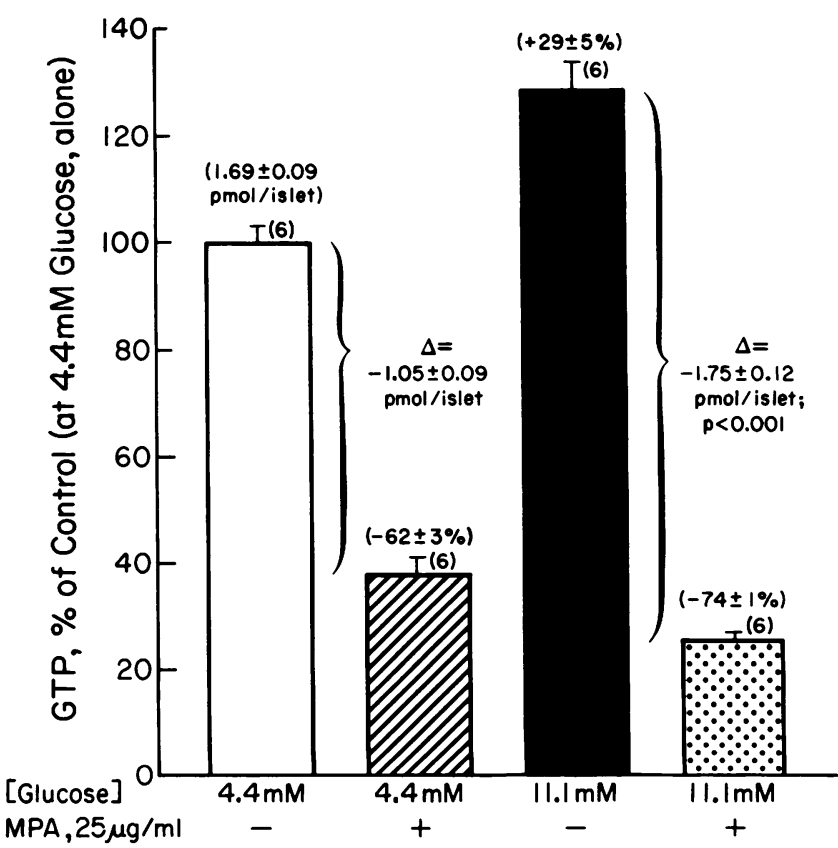

Figure 2. Glucose dependency of GTP content of islets and its inhibitibility by MPA. Bars are expressed as \% of a common reference value (4.4 mM glucose alone). Data are expressed as mean ( \pm SEM) for six determinations each, from two separate experiments showing identical results. The rise in GTP at $11.1 \mathrm{mM}$ glucose is statistically significant $(P<0.05$; not indicated in the Figure). 


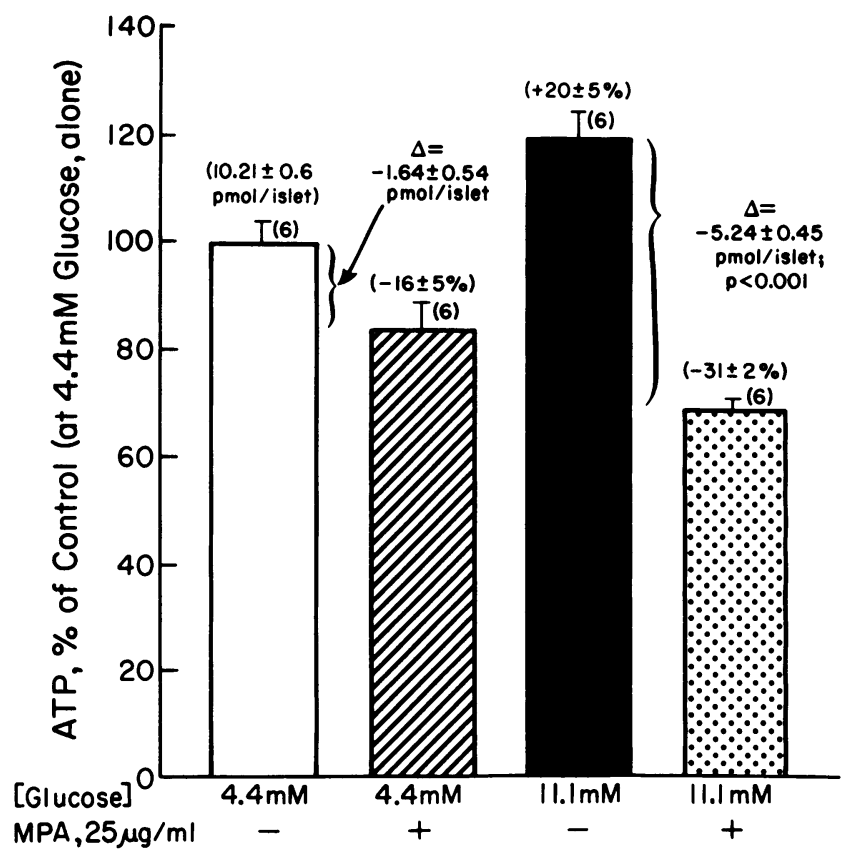

Figure 3. Glucose dependency of ATP content of islets and its inhibitibility by MPA. Bars are expressed as \% of a common reference value (4.4 mM glucose alone). Data are expressed as mean ( \pm SEM) for six determinations each, from two separate experiments showing identical results. The $20 \%$ rise in ATP at high glucose was not statistically significant within this study, but was seen in every experiment.

GTP content was now lower $(P<0.05)$ at high glucose after MPA (cf. Figs. 2 and 5).

The increment in both purine and pyrimidine nucleotides induced by glucose suggests that an elevation in the ambient glucose concentration might increase the content of an intermediate required in both pathways (possibly PRPP; see below). To test this possibility further, exogenous guanine was provided in order to fuel the "salvage" pathway by direct coupling to PRPP. When $75 \mu \mathrm{M}$ guanine was provided at $4.4 \mathrm{mM}$ glucose (in the absence of MPA), GTP content rose significantly (by $33 \%$, or by $0.65 \pm 0.06 \mathrm{pmol} /$ islet) to levels now similar to those seen at $11.1 \mathrm{mM}$ glucose alone (Fig. 5), whereas, in contrast, no significant further rise in GTP (Fig. 5) or ATP was seen when guanine was provided at $11.1 \mathrm{mM}$ glucose (Fig. 5). However, when guanine ( $75 \mu \mathrm{M})$ was provided in the presence of MPA, islet content of GTP rose $65 \%$ more, and reached higher final levels $(2.24 \pm 0.13$ vs. $2.99 \pm 0.22 ; P$ $<0.05)$ at the higher glucose concentration despite again starting from a lower absolute level $(P<0.05)$ after MPA treatment (Fig. 5). Likewise, guanine increased ATP content at $11.1 \mathrm{mM}$ glucose only in the presence of MPA; ATP rose from $7.32 \pm 0.33$ to $11.5 \pm 0.16 \mathrm{pmol} /$ islet $(P<0.01)$. These findings support the formulation that a regulatory precursor (presumably PRPP) does indeed rise with high glucose levels but is shunted into IMP-dependent pathways; however, when this preferred pathway (s) is rendered unavailable due to the block imposed by MPA, the metabolite becomes free to fuel the guanine-induced "salvage" synthesis of GTP as well (cf. references 27 and 28).

Effects of glucose, MPA, and/or guanine on UTP content. The formulation above is supported by measurements of UTP. MPA has been observed to induce a rise in cellular UTP content $(1,21,29)$ attributable to an increase in the availability of $\operatorname{PRPP}^{2}(1,27,28,30,31)$ which is then shunted into the pyrimidine synthetic pathway. Therefore, we quantitated UTP during the current studies to assess indirectly whether glucose increases availability of precursors for NTP synthesis. A reduction in glucose concentration (from 4.4 to $1.7 \mathrm{mM}$ ) or the addition of mannoheptulose to $4.4 \mathrm{mM}$ glucose reduced UTP in parallel to the changes in GTP and ATP (Table II). Reciprocally, increasing glucose from 4.4 to $11.1 \mathrm{mM}$ glucose nearly doubled UTP (Fig. 4; Table III). In the presence of MPA, UTP levels rose by similar amounts at both glucose concentrations

2. PRPP content reflects changes in both its synthesis and consumption. MPA disinhibits PRPP synthesis $(11,31)$ by reducing purine nucleotide synthesis. In addition, IMP, which rises $(23,28)$ proximal to the MPA-induced block at IMP dehydrogenase (32), can be degraded to inosine $(33,34)$ which in islets $(35)$ as in other tissues $(4,28,31)$ is degraded to ribose-1-phosphate (36). Ribokinase action converts ribose-1-phosphate to ribose-5-phosphate, which could increase synthesis of PRPP $(33,36)$, which, in turn, is utilized for UTP synthesis.

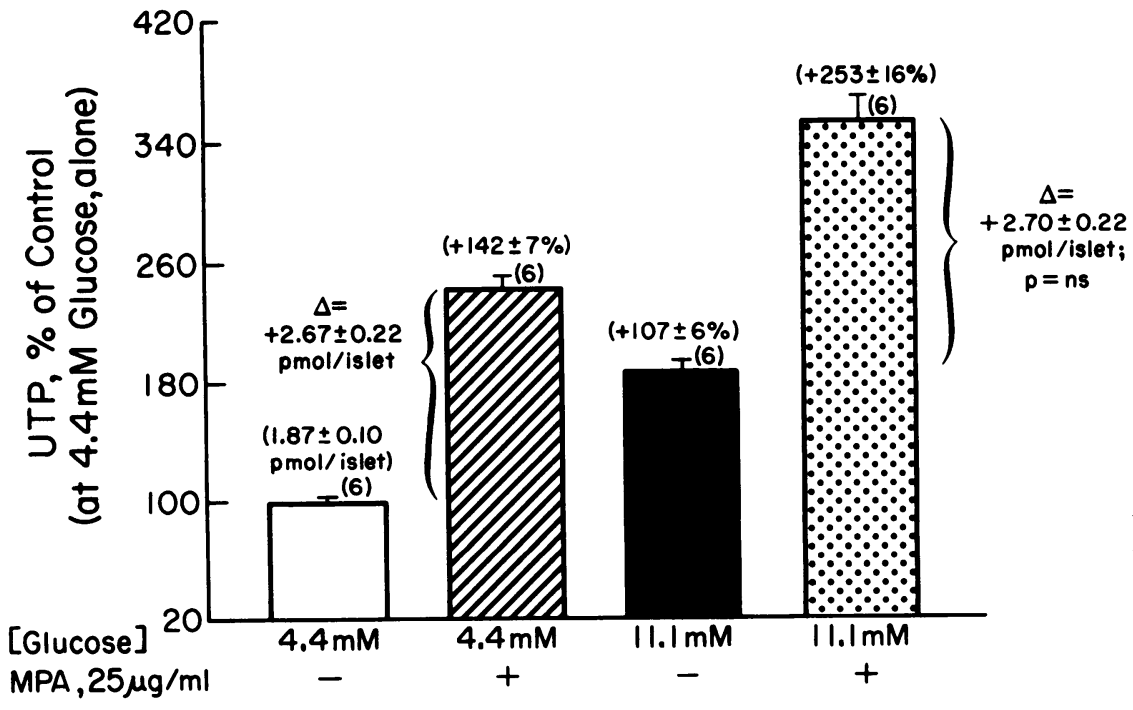

Figure 4. Glucose dependency of UTP content of islets and its stimulation by MPA. Bars are expressed as \% of a common reference value (4.4 $\mathrm{mM}$ glucose alone). Data are expressed as mean $( \pm$ SEM $)$ for six determinations each, from two separate experiments showing identical results. 


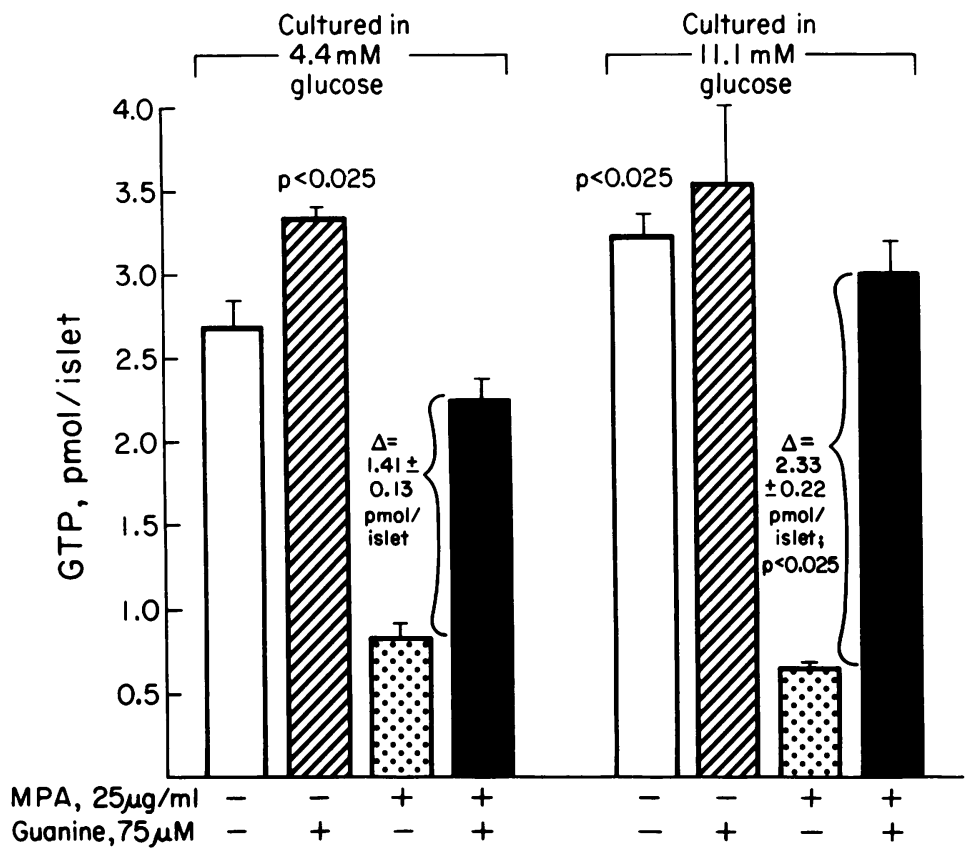

Figure 5. Glucose dependency of the GTP content of islets, in the presence and absence of MPA $(25 \mu \mathrm{g} / \mathrm{ml})$ or guanine $(75 \mu \mathrm{M})$. High glucose ( $11.1 \mathrm{mM})$ increased GTP compared to $4.4 \mathrm{mM}$ glucose (open bars); exogenously-provided guanine by itself (cross-hatched bars) increased GTP significantly only at the lower glucose concentration. Effects of MPA are shown (stippled bars) as is the addition of guanine to MPA (solid bars). Values are mean ( \pm SEM) for 2-3 determinations each. but again reached a higher final level at $11.1 \mathrm{mM}$ glucose $(P$ $<0.001$; Fig. 4 ; and Table III, Experiment 1 vs. 2); conversely, exogenous guanine (which consumes PRPP in the salvage pathway), reduced the UTP levels achieved in the presence of MPA ( Table III) towards control values (no MPA) ( Table III). Thus, provision of guanine largely reversed the effects of MPA on UTP, as it did on GTP and ATP content (reference 1; Figs. 2 and 3). To summarize, glucose and MPA both increase, and guanine decreases, UTP (probably due to changes in both PRPP synthesis and consumption; see Discussion).

Effect of guanine on insulin release. The effect on insulin release of guanine supplementation of the culture medium was also examined ( Table IV). By itself, guanine (30-100 $\mu \mathrm{M}$ ) had little effect on subsequent secretion after culture, whether provided at 4.4 or $11.1 \mathrm{mM}$ glucose (Table IV). In contrast, in the presence of MPA, guanine increased (i.e., restored) insulin release in proportion to the degree of inhibition of GTP content and insulin release caused by MPA, i.e., to a large degree at 11.1 $\mathrm{mM}$ glucose and minimally, if at all, at $4.4 \mathrm{mM}$ glucose (Table IV). Thus, guanine, via the salvage pathway, reverses the deficiency of pools of GTP involved in exocytosis, which had been inhibited by MPA.

Table II. Effect* of Glucose Concentration and Mannoheptulose on NTP Contents of Pancreatic Islets After an Overnight Culture

\begin{tabular}{|c|c|c|c|c|}
\hline Condition & GTP & ATP & UTP & Total NTP \\
\hline $\begin{array}{l}\text { A. Control }(4.4 \mathrm{mM} \\
\text { glucose) } \\
\text { B. } 4.4 \mathrm{mM} \text { glucose }\end{array}$ & $2.10 \pm 0.07$ & $7.83 \pm 0.24$ & $1.42 \pm 0.05$ & $11.36 \pm 0.33$ \\
\hline $\begin{array}{l}+3 \mathrm{mM} \\
\text { mannoheptulose }\end{array}$ & $\begin{array}{c}1.71 \pm 0.11 \\
(-19 \%)\end{array}$ & $\begin{array}{c}6.35 \pm 0.31 \\
(-19 \%)\end{array}$ & $\begin{array}{c}1.74 \pm 0.11 \\
(-17 \%)\end{array}$ & $\begin{array}{c}9.11 \pm 0.48 \\
(-19 \%)\end{array}$ \\
\hline C. $1.7 \mathrm{mM}$ glucose & $\begin{array}{c}1.74 \pm 0.11 \\
(-17 \%)\end{array}$ & $\begin{array}{c}6.76 \pm 0.41 \\
(-14 \%)\end{array}$ & $\begin{array}{c}1.09 \pm 0.07 \\
(-23 \%)\end{array}$ & $\begin{array}{l}9.60 \pm 0.57 \\
(-15 \%)\end{array}$ \\
\hline
\end{tabular}

\footnotetext{
* Values represent mean $( \pm$ SEM) of three determinations each. Comparison of experimental conditions $(B)$ or $(C)$ vs. their respective controls $(A)$ were statistically significant $(P<0.05$ or greater) for each nucleotide.
}

Radiolabeling of de novo or salvage GTP synthesis (Table V). These results together suggested that glucose augments flux into MPA-sensitive (i.e., IMP-derived) pool(s) of GTP. However, since IMP can be derived via several pathways, we ascertained directly whether de novo and salvage pathways contribute to GTP and ATP synthesis in islets. Purine nucleotide pools were labeled using $\left[{ }^{14} \mathrm{C}\right]$ glycine (which is incorporated into nucleotides specifically via the de novo pathway) or $\left[{ }^{3} \mathrm{H}\right]-$ hypoxanthine (an index of salvage pathway activity), under conditions (see "Methods") essentially identical to those used to ascertain the bulk content (mass) of ATP/GTP in islets. Glucose progressively augmented the labeling of both nucleotides using either precursor (Fig. 6). High glucose (11.1 mM) increased the areas under the curve for the GTP peak to 208 and $331 \%$ of values at 4.4 and $1.7 \mathrm{mM}$ glucose, respectively (labeled glycine) and to 190 and $279 \%$ (labeled hypoxanthine); corresponding values for ATP were 215 and $307 \%$ (labeled glycine) and 174 and 353\% (labeled hypoxanthine) (Table $\mathrm{V}$ and Fig. 6). Similar changes were seen in the specific activities (dpm/pmol) of GTP or ATP (Table V).

\section{Discussion}

Choice and relevance of experimental model. These studies were designed to determine whether islet GTP content, which has a permissive effect in exocytotic insulin release (1), is modified by the ambient glucose level. The experimental paradigm is based on the specific inhibition by MPA (or mizoribine) of IMP-dependent GTP synthesis, as assessed by steady-state GTP content (the biologically relevant parameter; reference 1). These drugs (8-10,18-20) block the conversion of IMP to xanthosine monophosphate (XMP), possibly also reinforcing this effect by inhibiting conversion of XMP to guanine nucleotides (19-21), but without any direct effects on other nucleotide pathways (20-24). RPMI 1640 was selected as the culture medium since it is purine-free (thus permitting manipulation of salvage pathway activity) and has an adequate concentration of inorganic phosphate $(5.6 \mathrm{mM})$, which is a regulatory 
Table III. Effect of Glucose Concentration During Culture, in the Presence and Absence of MPA $(25 \mu \mathrm{g} / \mathrm{ml})$ and Guanine $(75 \mu M)$, on UTP Content of Pancreatic Islets

\begin{tabular}{|c|c|c|}
\hline Condition & $\mathbf{L}$ & TP, pmol/islet* \\
\hline \multicolumn{3}{|l|}{ Experiment 1 . MPA absent } \\
\hline a. $4.4 \mathrm{mM}$ glucose & $1.92 \pm 0.05$ & $\Delta=1.51 \mathrm{pmol} /$ islet: \\
\hline b. $11.1 \mathrm{mM}$ glucose & $3.43 \pm 0.19\}$ & df $4 ; P<.01$ \\
\hline c. $4.4 \mathrm{mM}$ glucose + guanine & $2.12 \pm 0.08$ & $\Delta=0.78 \mathrm{pmol} /$ islet: \\
\hline d. $11.1 \mathrm{mM}$ glucose + guanine & $2.90 \pm 0.40$ & df $4 ; n=\mathrm{ns}$ \\
\hline \multicolumn{3}{|l|}{ Experiment 2. MPA present throughout } \\
\hline \multirow{4}{*}{$\begin{array}{l}\text { a. } 4.4 \mathrm{mM} \text { glucose + MPA } \\
\text { b. } 11.1 \mathrm{mM} \text { glucose + MPA } \\
\text { c. } 4.4 \mathrm{mM} \text { glucose + MPA + guanine } \\
\text { d. } 11.1 \mathrm{mM} \text { glucose + MPA + guanine }\end{array}$} & & \\
\hline & $\left.\begin{array}{l}4.59 \pm 0.36 \\
602+0.14\end{array}\right\}$ & $\Delta=1.43 ;$ df $3 ; P<.05$ \\
\hline & $6.02 \pm 0.14]$ & \\
\hline & $\left.\begin{array}{l}2.60 \pm 0.19^{+} \\
4.31 \pm 0.17^{\S}\end{array}\right\}$ & $\Delta=1.71 ;$ df $3 ; P<.01$ \\
\hline
\end{tabular}

* Values are the mean $( \pm \mathrm{SEM}) .{ }^{\ddagger} P<0.02$ vs. guanine-free values. ${ }^{\S} P<0.005$ vs. guanine-free values.

cofactor for synthesis of PRPP and of purine nucleotides (12, 37-40). 10\% undialyzed FBS was provided as a potential source $(33,41,42)$ of small amounts of hypoxanthine, in order to mimic the presence of the low amounts of hypoxanthine circulating in blood (43-45). This amount of hypoxanthine supports a basal production of salvage pathway-derived nucleotides and thereby provides a physiologic restraint on the de novo pathway (41) without which the visualization of a regulatory role for PRPP concentration in de novo pathway activity might be obfuscated (33). Additionally, a narrow range of potentially relevant glucose concentrations was studied. However, as with any in vitro study, extrapolation of these findings to the situation in vivo should be made with caution.

Effects of MPA on GTP content and insulin release. In our previous studies at $11.1 \mathrm{mM}$ glucose, a saturating concentration of MPA $(25 \mu \mathrm{g} / \mathrm{ml})$ reduced GTP by $\sim 80 \%$ and glucoseinduced insulin release by $54 \pm 2 \%$. The current studies confirm these observations at nearly-normal glucose levels $(7.8 \mathrm{mM})$ during the antecedent overnight culture period. A central new observation was that the inhibition of insulin release induced by MPA (or mizoribine) declined steeply at only slightly lower glucose levels, being half-maximal at $6.4 \mathrm{mM}$ glucose and virtually absent at $4.4 \mathrm{mM}$. These studies suggest that even modest elevations in ambient glucose concentrations sensitize islets to inhibition by structurally dissimilar, selective inhibitors of IMP dehydrogenase. These findings imply that high glucose acts as a biologic "switch" to shunt nucleotide precursors preferentially into IMP-dependent pathways, rendering islet GTP content more susceptible to inhibition by the blockers. One might question whether the markedly blunted inhibition by MPA of insulin secretion seen after islet culture at lower glucose levels could be attributed to the relatively modest differences in the reduction of GTP achieved by MPA (inhibition of GTP after $11.1 \mathrm{mM}$ glucose $=80 \%$; inhibition of GTP after 4.4 $\mathrm{mM}$ glucose $=63 \%$ ). However, this does indeed seem to be the case, since this effect of $25 \mu \mathrm{g} / \mathrm{ml}$ MPA at $4.4 \mathrm{mM}$ glucose is

Table IV. Effects of MPA $(25 \mu \mathrm{g} / \mathrm{ml})$ in the Absence and Presence of Guanine, During Overnight Culture at 4.4 or $11.1 \mathrm{mM}$ Glucose, on Subsequent Insulin Secretion*

Incremental insulin response, $\mu \mathrm{U} \cdot 45 \mathrm{~min}$

I. Culture at $4.4 \mathrm{mM}$ glucose
a. Control (5)
b. MPA (5)
c. MPA $+75 \mu \mathrm{M}$ guanine (5)
d. $75 \mu \mathrm{M}$ guanine alone (5)

II. Culture at $11.1 \mathrm{mM}$ glucose
a. Control (5)
b. MPA (5)
c. MPA $+75 \mu \mathrm{M}$ guanine (4)
d. $75 \mu \mathrm{M}$ guanine alone (3)

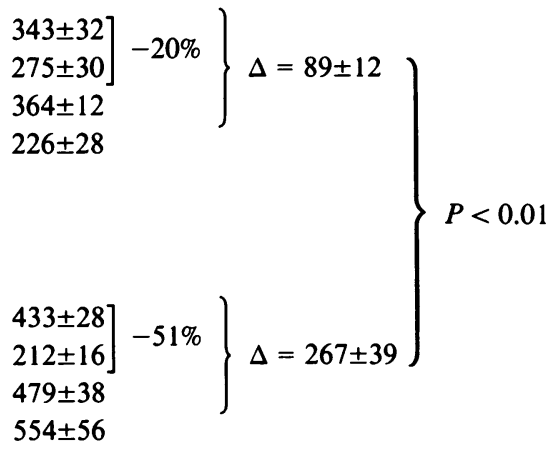

* Numbers in parantheses are the numbers of observations. Data are mean \pm SEM. 


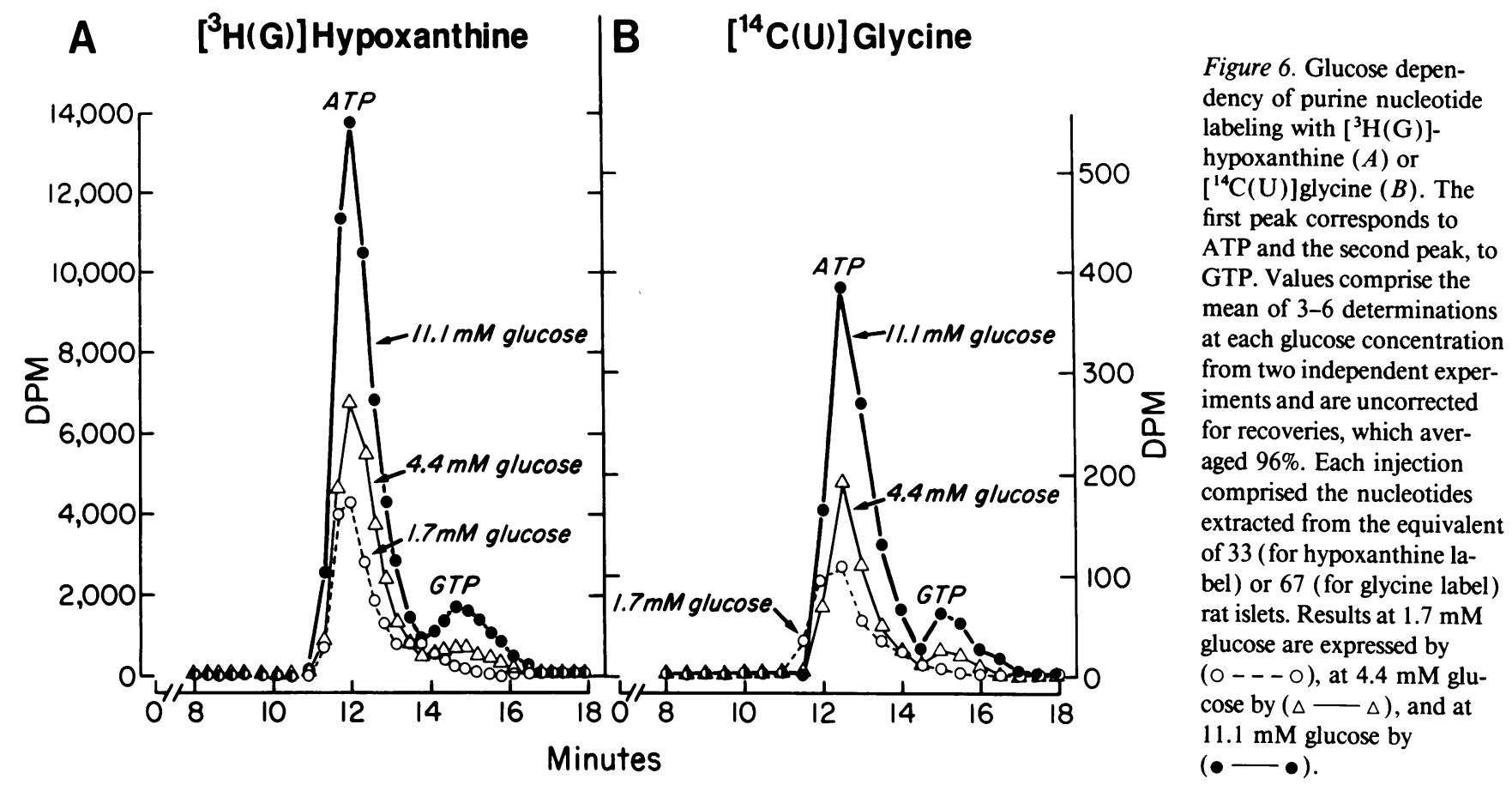

nearly superimposable on the effect of a much lower concentration $(0.5 \mu \mathrm{g} / \mathrm{ml})$ of MPA provided at $11.1 \mathrm{mM}$ glucose (GTP: $-60 \pm 2 \%$; insulin secretion; $-12 \%$ ) in our previous studies (1). In other words, MPA is only $\sim 1 / 50$ th as potent on exocytotic secretion at low as compared to higher glucose concentrations. This is because insulin release is not inhibited until GTP content falls (by $>80 \%$ ) to a level of $\leq 0.6-0.8 \mathrm{pmol} /$ islet (1), but falls off precipitously thereafter. Thus, the lesser effect of MPA on insulin release after culture in lower glucose concentrations reflects a different origin (and therein a lesser inhibitability by MPA) of the GTP content. However, not only GTP derived via IMP but also that derived via the guanine salvage pathway is capable of supporting secretion, since exogenous guanine restores basal- and glucose-stimulated GTP levels in MPA-treated islets (current data) and, concomitantly, reverses the effect of MPA to inhibit insulin secretion (current data and reference 1). Thus, these studies provide no evidence of a functional compartmentation of (MPA-sensitive vs. MPAinsensitive) cytosolic GTP pools with regard to exocytosis.

Effects of glucose on nucleotide content. Increasing glucose levels modestly but consistently augmented total islet GTP content (by $40-50 \%$, between 1.7 and $11.1 \mathrm{mM}$ glucose). These findings are in accord with studies of Zunkler et al. (46), Hoenig and Matschinsky (47), and Meglasson et al. (48) who noted small changes in GTP content (or GTP/GDP ratio) within 30-150 min of marked increases in the glucose concentration to which pancreatic islets were exposed. Glucose also slightly (but again consistently) increased ATP levels and dramatically increased UTP levels. The most parsimonious explanation for these findings is that glucose increases the availability of PRPP, the precursor common to all three purine or pyrimidine nucleotides. Since the $S_{0.5}$ (PRPP) for the de novo purine synthetic pathway is higher than the $K_{\mathrm{m}}$ (PRPP) in the pyrimidine synthetic and purine salvage pathways $(11,12,31$, $32,37,38,49$ ), and since PRPP availability is probably regulatory for all three $(11,41,50,51)$, an increment in PRPP might indeed be expected to be utilized by the UTP pathway and by HGPRTase first $(36,41)$, followed by the de novo synthetic pathway. Additionally, it is energetically reasonable (11) that salvage pathways would be preferred $(41,52)$ at lower levels of fuel availability $(27,36)$, since, unlike the de novo ${ }^{3}$ pathway, it does not directly consume ATP. Effects of glucose on NTP content were seen not only upon increasing glucose concentration, but also, upon decreasing it or upon impeding glucose phosphorylation through the use of mannoheptulose. The latter findings, in conjunction with the lack of a stimulatory effect of 3-O-methylglucose, demonstrate that the glucose increases NTP content only after its metabolism, perhaps to ribose-5-phosphate (the precursor of PRPP).

The conclusion that glucose preferentially shunts GTP production from IMP-independent pathways to IMP-dependent pathwars is supported by four findings: First, MPA, a selective inhibitor of IMP dehydrogenase, reduced GTP content more at higher glucose levels. Second, MPA prevented the glucose-induced increment in GTP (and ATP). Third, the residual GTP remaining after MPA exposure ${ }^{4}$ was consistently smaller at higher glucose concentrations. Lastly, exogenous provision of guanine ( to fuel the salvage pathways) stimulated a greater rise in GTP at higher glucose levels, but only when flux from IMP to GTP was prevented by treatment with MPA. In contrast, the

3. In additional studies, we have documented that at the glucose levels studied, hypoxanthine salvage contributes more to the mass of GTP and ATP in islets than does the de novo synthetic pathway (Meredith, M., M. Rabaglia, and S. Metz, manuscript in preparation).

4. This pool of GTP might reflect several potential sources of GTP derived via transphosphorylation or substrate (GDP) level phosphorylations (including nucleoside diphosphokinase, succinate thiokinase, and phosphoenolpyruvate carboxykinase) as well as some salvage of endogenously-produced guanine. Note that nucleoside diphosphokinase (reference 46 and Kowluru, A., and S. Metz, unpublished observations) and succinate thiokinase (53), but not PEP carboxykinase (54), are present in pancreatic islets. 


\begin{tabular}{|c|c|c|c|c|}
\hline & \multicolumn{2}{|c|}{ Specific activity, dpm/pmol } & \multicolumn{2}{|c|}{$\begin{array}{l}\text { Absolute areas under } \\
\text { the peaks, total } \mathrm{dpm}^{\ddagger}\end{array}$} \\
\hline & GTP & ATP & GTP & ATP \\
\hline \multicolumn{5}{|l|}{$\begin{array}{l}\text { A. }\left[{ }^{14} \mathrm{C}(\mathrm{U})\right] \text { glycine label } \\
\text { (de novo synthesis) }\end{array}$} \\
\hline i. $1.7 \mathrm{mM}$ glucose & $0.45 \pm 0.02$ & $0.78 \pm 0.01$ & $57 \pm 3$ & $359 \pm 8$ \\
\hline ii. $4.4 \mathrm{mM}$ glucose & $0.70 \pm 0.04$ & $1.15 \pm 0.10$ & $90 \pm 6$ & $512 \pm 38$ \\
\hline iii. $11.1 \mathrm{mM}$ glucose & $1.22 \pm 0.09$ & $2.06 \pm 0.06$ & $187 \pm 14$ & $1103 \pm 36$ \\
\hline \multicolumn{5}{|c|}{$\begin{array}{l}\text { B. }\left[{ }^{3} \mathrm{H}(\mathrm{G})\right] \text { hypoxanthine label } \\
\text { (salvage pathway) }\end{array}$} \\
\hline i. $1.7 \mathrm{mM}$ glucose & $66 \pm 3.18^{8}$ & $78 \pm 4.26$ & $3,645 \pm 30$ & $16,172 \pm 569$ \\
\hline ii. $4.4 \mathrm{mM}$ glucose & $70 \pm 3.61^{\S}$ & $124 \pm 0.88$ & $5,374 \pm 231$ & $32,817 \pm 2491$ \\
\hline iii. $11.1 \mathrm{mM}$ glucose & $132 \pm 9.56$ & $219 \pm 2.17$ & $10,186 \pm 679$ & $57,096 \pm 1195$ \\
\hline
\end{tabular}

\footnotetext{
*Values are expressed as specific activity (dpm/pmol) or areas under the peaks; mean ( \pm SEM) for 2-6 determinations each from two independent experiments. All comparisons for ATP or GTP between different glucose levels are statistically significant $(P<0.05$ or greater level of significance) except for the single comparison indicated by ${ }^{\S}$ (hypoxanthine labeling of GTP at $1.7 \mathrm{vs.} 4.4 \mathrm{mM}$ glucose). $\quad \neq 200$ islets per condition were cultured overnite at the indicated glucose concentrations in the presence of $10 \mu \mathrm{Ci} / \mathrm{ml}\left[{ }^{14} \mathrm{C}(\mathrm{U})\right] \mathrm{glycine}$ or $2 \mu \mathrm{Ci} / \mathrm{ml}$ $\left[{ }^{3} \mathrm{H}(\mathrm{G})\right]$ hypoxanthine, and then were extracted. An aliquot of the supernatant (equivalent to the content of 66 islets for glycine labeling and of 33 islets for hypoxanthine labeling) was analyzed by HPLC and expressed as total counts under the corresponding curves for data analysis.
}

exogenous provision of guanine did not cause an increment in GTP content at high glucose levels in the absence of MPA (i.e., where feedback inhibition of the salvage pathway by endogenous nucleotides was maximally operative); this indicates, in addition, that glucose probably does not directly stimulate guanine uptake or salvage pathway activity. These observations indirectly support the formulation suggested above that glucose might increase PRPP levels which, in the absence of MPA, is funneled preferentially by glucose, first into pyrimidine (UTP) synthesis, and then into the hypoxanthine salvage and de novo pathways. Rigorous study of the fluxes through the biochemical pathways involved, and direct measurements of PRPP availability, will be required to substantiate or refute these tentative conclusions, since it cannot be assumed that physiologic changes in glucose metabolism would necessarily increase PRPP synthesis. However, this formulation is reinforced by the studies of Pilz et al. using human lymphocytes (27), in which they observed that increasing extracellular glucose concentrations augmented intracellular ribose-5-phosphate and PRPP concentrations, promoted de novo purine nucleotide synthesis, and rendered the nucleotide content of the cells more susceptible to blockade by inhibitors of de novo synthetic pathways. These investigators noted that inhibitors of de novo GTP synthesis further augment PRPP availability ${ }^{2}$; if this occurs in islets, it would explain the additive effects of high glucose and MPA on UTP content of islets.

Relationship between GTP content and insulin secretion. In our studies, modest increments in GTP content induced by guanine at $4.4 \mathrm{mM}$ glucose (to levels identical to those seen at $11.1 \mathrm{mM}$ glucose alone; Fig. 5) had little effect on subsequent insulin release, suggesting that GTP levels must be close to saturating for secretion at physiologic glucose concentrations. In fact, a reduction of up to $60 \%$ in GTP content induced by submaximal concentrations of MPA (up to $0.1-1 \mu \mathrm{g} / \mathrm{ml}$ ) at $11.1 \mathrm{mM}$ glucose (1) do not detectably impair subsequent secretion. However, when GTP content is inhibited more (see above) there is a steep decline in subsequent insulin secretory responses (and, reciprocally, a considerable rise in both GTP and insulin release when exogenous guanine is provided). Thus, under physiologic conditions, glucose availability may never become limiting for the GTP involved in insulin secretion (leading us to define its role as "permissive" only; reference 1). However, similar studies need to be carried out with regard to other glucose-dependent effects of purine nucleotides; for example, purine nucleotides are utilized for RNA synthesis (6) and DNA synthesis (7), which in turn are needed for islet protein synthesis and beta cell proliferation.

The rise in GTP (and in insulin secretion) induced by guanine, and the fall in both parameters induced by MPA, were in general, inversely proportional to the starting content of purine nucleotides, which are feedback inhibitors of salvage (11) and de novo $(11,31,55)$ synthetic pathways as well as of the synthesis of their common precursor PRPP $(11,24,41,55-57)$. Thus, our studies overall are quite compatible with data from other cells, which suggest that the cellular content of NTP is modulated by two interactive variables - the negative feedback control of nucleotides upon their own synthesis (at several enzymatic levels; references $11,24,31,55-58$ ) and positive regulatory control by PRPP $(27,38,41,56)$.

Possible site(s) of action of glucose on nucleotide synthesis. We hypothesized that glucose shunts substrates such as PRPP into IMP; however, it was unknown whether the two major pathways potentially yielding IMP (the de novo and salvage pathways ) actually are present in islets. Therefore, we assessed their presence in islets via, respectively, the incorporation of $\left[{ }^{14} \mathrm{C}\right]$ glycine or $\left[{ }^{3} \mathrm{H}\right]$ hypoxanthine into nucleotides. Our studies indicate that both pathways can generate ATP and GTP in islets and that glucose augments both their activities. The effect of glucose is even more impressive when one considers that glucose inhibits the uptake of glycine into islets (59). Such labeling studies cannot be used to quantitate precisely the relative contributions ${ }^{3}$ of the two pathways to the abundance (mass) of ATP or GTP measured in islets; however, the considerably greater effect of glucose on the specific activity of GTP 
and/or ATP, compared to its effect on their total mass, suggests that these two pathways may, in fact, be selectively activated by glucose. This finding can be utilized profitably in future studies to assess more sensitively any acute effects of glucose on ATP or GTP synthesis.

\section{Acknowledgments}

The technical assistance of Thom Rabaglia and Kenneth Finn are gratefully acknowledged.

These studies were funded in part by the Veterans Administration, and the National Institutes of Health (grant DK 37312).

\section{References}

1. Metz, S. A., M. E. Rabaglia, and T. J. Pintar. 1992. Selective inhibitors of GTP synthesis impede exocytotic insulin release from intact rat islets. $J$. Biol. Chem. 267:12517-12527.

2. Bergsten, P. 1987. Significance of the calcium content of mouse $\beta$ cells in the preservation of glucose-induced insulin release during culture. J. Endocrinol. 115:27-34.

3. Andersson, A. 1978. Isolated mouse pancreatic islets in culture. Effects of serum and different culture media on insulin production of islets. Diabetologia. 14:397-404.

4. Morris, R. E., J. Wang, J. R. Blum, T. Flavin, M. P. Murphy, S. J. Almquist, N. Chu, Y. L. Tam, M. Kaloostian, A. C. Allison, and E. M. Eugui. 1991. Immunosuppressive effects of morpholinoethyl ester of mycophenolic acid ( RS-61443) in rat and nonhuman primate recipients of heart allografts. Transplant. Proc. 23:19-25.

5. Sollinger, H. W., M. Deierhoi, F. O. Belzer, A. Diethelm, and R. Kauffman. 1992. RS-61443: Phase 1 clinical trial and pilot rescue study. Transplantation (Baltimore). 53:428-432.

6. Welsh, N., S. Sandler, M. Welsh, and C. Hellerström. 1986. Regulation of RNA metabolism in relation to insulin production and oxidative metabolism in mouse pancreatic islets in vitro. Biochim. Biophys. Acta. 887:58-68.

7. Liang, Y., H. Najafi, R. M. Smith, E. C. Zimmerman, M. A. Magnuson, M. Tal, and F. M. Matschinsky. 1992. Concordant glucose induction of glucokinase, glucose usage, and glucose-stimulated insulin release in pancreatic islets maintained in organ culture. Diabetes. 41:792-806.

8. Sakaguchi, K., M. Tsujino, M. Yoshizawa, K. Mizuno, and K. Hayano. 1975. Action of bredinin on mammalian cells. Cancer Res. 35:1643-1648.

9. Koyama, H., and M. Tsuji. 1983. Genetic and biochemical studies on the activation and cytotoxic mechanism of bredinin, a potent inhibitor of purine biosynthesis in mammalian cells. Biochem. Pharmacol. 32:3547-3553.

10. Turka, L. A., J. Dayton, G. Sinclair, C. B. Thompsom, and B. S. Mitchell. 1991. Guanine nucleotide depletion inhibits T cell activation. J. Clin. Invest. 87:940-948.

11. Henderson, J. F., and A. R. P. Paterson. 1973. In Nucleotide Metabolism: An Introduction. Chapters 6-8. Academic Press, New York. 80-135.

12. Holmes, E. W., J. A. McDonald, J. M. McCord, J. B. Wyngaarden, and W. N. Kelley. 1973. Human glutamine phosphoribosylpyrophosphate amidotransferase. J. Biol. Chem. 248:144-150.

13. Crabtree, G. W., and J. F. Henderson. 1971. Rate-limiting steps in the interconversion of purine ribonucleotides in Ehrlich ascites tumor cells in vitro. Cancer Res. 31:985-991.

14. Raivio, K. O., and J. E. Seegmiller. 1973. Role of glutamine in purine synthesis and in guanine nucleotide formation in normal fibroblasts and in fibroblasts deficient in hypoxanthine phosphoribosyltransferase activity. Biochim. Biophys. Acta. 299:283-292.

15. Metz, S. A. 1985. Glucose increases the synthesis of lipoxygenase-mediated metabolites of arachidonic acid in intact rat islets. Proc. Natl. Acad. Sci. USA. 82:198-202.

16. Metz, S. A., and M. Dunlop. 1990. Stimulation of insulin release by phospholipase D. Biochem. J. 270:427-435.

17. Metz, S. A. 1988. Exogenous arachidonic acid promotes insulin release from intact or permeabilized rat islets by dual mechanisms. Diabetes. 37:14531469.

18. Franklin, T. J., and J. M. Cook. 1969. The inhibition of nucleic acid synthesis by mycophenolic acid. Biochem. J. 113:515-524.

19. Sweeney, M. J., D. H. Hoffman, and M. A. Esterman. 1972. Metabolism and biochemistry of mycophenolic acid. Cancer Res. 32:1803-1809.

20. Snyder, F. F., J. F. Henderson, and D. A. Cook. 1972. Inhibition of purine metabolism-computer-assisted analysis of drug effects. Biochem. Pharmacol. 21:2351-2357.
21. Lee, H.-J., K. Pawlak, B. T. Nguyen, R. K. Robins, and W. Sadée. 1985 Biochemical differences among four inosinate dehydrogenase inhibitors, mycophenolic acid, ribavirin, tiazofurin, and selenazofurin, studied in mouse lymphoma cell culture. Cancer Res. 45:5512-5520.

22. Rizzo, M. T., G. Tricot, R. Hoffman, H. N. Jayaram, G. Weber, J. G. N. Garcia, and D. English. 1990. Inosine monophosphate dehydrogenase inhibitors Probes for investigations of the functions of guanine nucleotide binding proteins in intact cells. Cell Signalling. 2:509-519.

23. Kagawa, D., T. Nakamura, T. Ueda, S. Ando, H. Tsutani, M. Uchida, N. Domae, M. Sasada, and H. Uchino. 1986. Reverse effect of guanine on the inhibitory action of mycophenolic acid during nucleic acid synthesis. Anticancer Res. 6:643-648.

24. Barankiewicz, J., and J. F. Henderson. 1976. Effect of lowered intracellular ATP and GTP concentrations on purine ribonucleotide synthesis and interconversion. Can. J. Biochem. 55:257-262.

25. Ashcroft, S. J. H. 1981. Metabolic controls of insulin secretion. In The Islets of Langerhans: Biochemistry, Physiology, and Pathology. S. J. Cooperstein and D. Watkins, editors. Academic Press, New York. 117-148.

26. Zawalich, W. S. 1979. Intermediary metabolism and insulin secretion from isolated rat islets of Langerhans. Diabetes. 28:252-260.

27. Pilz, R. B., R. C. Willis, and G. R. Boss. 1984. The influence of ribose 5-phosphate availability on purine synthesis of cultured human lymphoblasts and mitogen-stimulated lymphocytes. J. Biol. Chem. 259:2927-2935.

28. Boss, G. R. 1984. Decreased phosphoribosylpyrophosphate as the basis for decreased purine synthesis during amino acid starvation of human lymphoblasts. J. Biol. Chem. 259:2936-2941.

29. Cohen, M. B., and W. Sadée. 1983. Contributions of the depletions of guanine and adenine nucleotides to the toxicity of purine starvation in the mouse T lymphoma cell line. Cancer Res. 43:1587-1591.

30. Lui, M. S., M. A. Faderan, J. J. Liepnieks, Y. Natsumeda, E. Olah, H. N. Jayaram, and G. Weber. 1984. Modulation of IMP dehydrogenase activity and guanylate metabolism by tiazofurin (2- $\beta$-ribofuranosylthiazole-4-carboxamide. J. Biol. Chem. 259:5078-5082.

31. Bagnara, A. S., A. A. Letter, and J. F. Henderson. 1974. Multiple mechanisms of regulation of purine biosynthesis de novo in intact tumor cells. Biochim Biophys. Acta. 374:259-270.

32. Wood, A. W., M. A. Becker, and J. E. Seegmiller. 1973. Purine nucleotide synthesis in lymphoblasts cultured from normal subjects and a patient with Lesch-Nyhan Syndrome. Biochem. Genet. 9:261-274.

33. Hershfield, M. S., and J.E. Seegmiller. 1977. Regulation of de novo purine synthesis in human lymphohlasts. J. Biol. Chem. 252:6002-6010.

34. Brosh, S., O. Sperling, E. Dantziger, and Y. Sidi. 1992. Metabolism of guanine and guanine nucleotides in primary rat neuronal cultures. J. Neurochem. 58:1485-1490

35. Capito, K., and C. J. Hedeskov. 1976. Inosine-stimulated insulin release and metabolism of inosine in isolated mouse pancreatic islets. Biochem. $J$. 158:335-340.

36. Barankiewicz, J., and J. F. Henderson. 1977. Ribose 1-phosphate metabolism in Ehrlich ascites tumor cells in vitro. Biochim. Biophys. Acta. 479:371-377.

37. Kim, Y.-A., M. T. King, W. E. Teague, Jr., G. A. Rufo, Jr., R. L. Veech, and J. V. Passonneau. 1992. Regulation of the purine salvage pathway in rat liver. Am. J. Physiol. 262:E344-E352.

38. Becker, M. A., K. O. Raivio, and J. E. Seegmiller. 1979. Synthesis of phosphoribosylpyrophosphate in mammalian cells. Adv. Enzymol. Relat. Areas. Mol. Biol. 49:281-306.

39. Hershko, A., A. Razin, and J. Mager. 1969. Regulation of the synthesis of ${ }_{5}$-phosphoribosyl-,-pyrophosphate in intact red cells and in cell-free preparations. Biochim. Biophys. Acta. 184:64-76.

40. Boer, P., B. Lipstein, A. De Vries, and O. Sperling. 1976. The effect of ribose-5-phosphate and 5-phosphoribosyl-1-pyrophosphate availability on de novo synthesis of purine nucleotides in rat liver slices. Biochim. Biophys. Acta. 432:10-17.

41. Gordon, R. B., L. Thompson, L. A. Johnson, and B. T. Emmerson. 1979. Regulation of purine de novo synthesis in cultured human fibroblasts: The role of P-ribose-PP. Biochim. Biophys. Acta. 562:162-176.

42. Bökkerink, J. P. M., M. A. H. Bakker, T. W. Hulscher, R. R. A. De Abreu, E. D. A. M. Schretlen, J. P. R. M. van Laarhoven, and C. H. M. M. De Bruyn. 1986. Sequence-, time- and dose-dependent synergism of methotrexate and 6-mercaptopurine in malignant human T-lymphoblasts. Biochem. Pharmacol. $35: 3549-3555$

43. Puig, J. G., M. L. Jiménez, F. A. Mateos, and I. H. Fox. 1989. Adenine nucleotide turnover in hypoxanthine-guanine phosphoribosyl-transferase deficiency: evidence for an increased contribution of purine biosynthesis de novo. Metab. Clin. Exp. 38:410-418.

44. Goldfinger, S., J. R. Klinenberg, and J. E. Seegmiller. 1965. The renal excretion of oxypurines. J. Clin. Invest. 44:623-628.

45. Howell, S. B., K. Herbst, G. R. Boss, and E. Frei III. 1980. Thymidine requirements for the rescue of patients treated with high-dose methotrexate. Cancer Res. 40:1824-1829. 
46. Zünkler, B. J., S. Lenzen, and U. Panten. 1986. D-glucose enhances GTP content in mouse pancreatic islets. IRCS Med. Sci. 14:354-355.

47. Hoenig, M., and F. M. Matschinsky. 1987. HPLC analysis of nucleotide profiles in glucose-stimulated perifused rat islets. Metab. Clin. Exp. 36:295-301

48. Meglasson, M. D., J. Nelson, D. Nelson, and M. Erecinska. 1989. Bioenergetic response of pancreatic islets to stimulation by fuel molecules. Metab. Clin. Exp. 38:1188-1195.

49. Natsumeda, Y., N. Prajda, J. P. Donohue, J. L. Glover, and G. Weber. 1984. Enzymic capacities of purine de novo and salvage pathways for nucleotide synthesis in normal and neoplastic tissues. Cancer Res. 44:2475-2479.

50. Becker, M. A., and M. Kim. 1987. Regulation of purine synthesis de novo in human fibroblasts by purine nucleotides and phosphoribosyl pyrophosphate. J. Biol. Chem. 262:14531-14537.

51. Yen, R. C. K. Y., K. O. Rarvio, and M. A. Becker. 1981. Inhibition of phosphoribosyl-pyrophosphate synthesis in human fibroblasts by 6-methylthioinosinate. J. Biol. Chem. 256:1839-1845.

52. Smith, C. M., and J. F. Henderson. 1976. Relative importance of alternative pathways of purine nucleotide biosynthesis in Ehrlich ascites tumor cells in vivo. Can. J. Biochem. 54:341-349.

53. Fahien, L. A., M. J. MacDonald, E. H. Kmiotek, R. J. Mertz, and C. M.
Fahien. 1988. Regulation of insulin release by factors that also modify glutamine dehydrogenase. J. Biol. Chem. 263:13610-13614.

54. MacDonald, M. J., D. I. McKenzie, T. M. Walker, and J. H. Kaysen. 1992. Lack of glyconeogenesis in pancreatic islets: expression of gluconeogenic enzyme genes in islets. Horm. Metab. Res. 24:158-160.

55. Henderson, J. F. 1962. Feedback inhibition of purine biosynthesis in ascites tumor cells. J. Biol. Chem. 237:2631-2635.

56. Becker, M. A., M. J. Losman, and M. Kim. 1987. Mechanisms of accelerated purine nucleotide synthesis in human fibroblasts with superactive phosphoribosylpyrophosphate synthetases. J. Biol. Chem. 262:5596-5602.

57. Thompson, L. F., R. C. Willis, J. W. Stoop, and J. E. Seegmiller. 1978. Purine metabolism in cultured human fibroblasts derived from patients deficient in hypoxanthine phosphoribosyltransferase, purine nucleoside phosphorylase, or adenosine deaminase. Proc. Natl. Acad. Sci. USA. 75:3722-3726.

58. Henderson, J. F., and M. K. Y. Khoo. 1965. Availability of 5-phosphoribosyl 1-pyrophosphate for ribonucleotide synthesis in Ehrlich ascites tumor cells in vitro. J. Biol. Chem. 240:2358-2362.

59. Sehlin, J. 1972. Transport and oxidation of glycine in mammalian pancreatic islets with reference to the mechanism of amino acid-induced insulin release. Hormones (Basel). 3:144-155. 